\title{
Bien écrire pour bien se faire comprendre : mise à jour des instructions aux auteurs
}

\author{
Marco L.A. Sivilotti, MD, MSc \\ LA VERSION FRANÇAISE DES INSTRUCTIONS AUX AUTEURS PARAÎTRA \\ DANS L'ÉDITION DE JANVIER 2008, VOL. 10, NO 1, DU JCMU. \\ ENGLISH VERSION OF THIS EDITORIAL ON PAGE 417
}

$\mathrm{L}$ e conseil de rédaction du JCMU annonce que les instructions aux auteurs ont été revues. Cette nouvelle reflète la qualité croissante des soumissions que nous recevons et notre objectif continu de publier des articles clairs et exacts apportant une contribution importante à la littérature médicale. L'arrivée de sang nouveau dans le bureau de rédaction a été l'occasion de mettre ses instructions à jour et, dans certains cas, d'élever les normes s'appliquant aux recherches originales.

La dualité de la forme et du contenu est un élément essentiel de la critique littéraire, des arts et de la rhétorique. Il faut néanmoins uniformiser le style et la structure de la littérature scientifique permanente si l'on veut maintenir l'accent sur le message. Le JCMU reconnaît ce principe depuis longtemps et il continue d'adhérer aux critères de l'énoncé «Uniform Requirements for Manuscripts Submitted to Biomedical Journals » de l'International Committee of Medical Journal Editors (ICMJE) ${ }^{1}$. En fait, la première phrase de la rubrique sur la présentation des manuscrits, qui renvoie le lecteur au site Web de l'ICMJE, demeure inchangée et constitue une excellente première étape pour tout auteur entreprenant la rédaction d'un manuscrit. Contrairement à l'opinion populaire, ces exigences traitent de beaucoup plus que les critères concernant les qualités d'auteur et la présentation des citations. Les auteurs chevronnés y trouveront également leur compte.

\section{Facilitation du processus d'évaluation par les pairs}

Les auteurs potentiels ne devraient toutefois pas s'arrêter là. Ils devraient lire les instructions attentivement et les consulter tout au long du processus de préparation de manuscrits. Bon nombre d'entre nous frissonnent à l'idée d'ordres préimprimés pour la prise en charge de patients asthmatiques, mais nous devons reconnaître que la normalisation du format des manuscrits facilite le processus d'évaluation par les pairs. En suivant ces instructions, les auteurs font preuve de respect envers les évaluateurs, ne leur faisant pas perdre leur temps. Ces derniers ont aussi appris que la rédaction négligée va de pair avec une démarche scientifique qui manque de rigueur. L'attention minutieuse avec laquelle la recherche est effectuée devrait se refléter dans la rédaction des rapports.

\section{Initiatives pour améliorer la qualité}

On trouve facilement une multitude de preuves que la rédaction scientifique a besoin de toute l'aide qu'on veut bien lui accorder ${ }^{2}$. Une gamme d'initiatives, telles que l'énoncé CONSORT (Consolidated Standards of Reporting Trials) pour la présentation d'essais cliniques randomisés et l'initiative STARD (Standards for Reporting of Diagnostic Accuracy) pour la présentation d'études sur l'exactitude du diagnostic, ont été élaborées non seulement pour normaliser la rédaction d'articles, mais aussi pour en améliorer la qualité et la clartés. Plus d'une décennie s'est écoulée depuis que Gilbert et ses collègues ont proposé des critères méthodologiques pour évaluer la qualité d'études rétrospectives de dossiers médicaux (c.-à-d. examens de dossiers médicaux $)^{4}$, mais l'intérêt suscité par ce concept populaire demeure faible 5 .

\section{Enregistrement des essais cliniques}

En plus de souscrire à ces initiatives, le $J C M U$ exige 
dorénavant l'enregistrement de tout essai clinique. Les chercheurs devraient noter que l'ICMJE définit un essai clinique comme suit : toute étude dans laquelle les participants ou les groupes de participants sont affectés, dès le départ, à une ou des interventions d'ordre sanitaire afin d'évaluer les effets de ces dernières sur la santé. Ces interventions peuvent porter non seulement sur des médicaments ou des dispositifs, mais aussi sur des actes chirurgicaux, des thérapies comportementales, des changements dans les protocoles de soins, et autres ${ }^{1}$.

Au final, cependant, l'obtention de l'imprimatur de ces listes de contrôle ne devrait nullement détourner les auteurs de leur objectif premier, à savoir la diffusion d'un message clair et convaincant au lectorat présent et futur. Le JCMU a toujours préconisé la précision et la clarté d'expression dans un style concis et direct. Bon nombre d'entre nous sont peut-être coupables de mettre l'accent sur l'observation de normes méthodologiques et les analyses statistiques rigoureuses au détriment de la langue, tant dans notre travail que dans nos revues. Les intéressés peuvent consulter d'excellentes monographies sur le sujet ${ }^{6-9}$. Nous imposerons leur lecture aux plus grands offenseurs. À bon entendeur, salut!

\section{Références}

1. International Committee of Medical Journal Editors. Uniform requirements for manuscripts submitted to biomedical journals: writing and editing for biomedical publication. Disponible sur : www.icmje.org/ (Consulté le 17 octobre 2007).

2. Altman DG. Poor-quality medical research: What can journals do? JAMA 2002;287:2765-7.

3. Altman DG for the CONSORT Group. Endorsement of the CONSORT statement by high impact medical journals: survey of instructions for authors. BMJ 2005;330:1056-7.

4. Gilbert EH, Lowenstein SR, Koziol-McLain J, et al. Chart reviews in emergency medicine research: Where are the methods? Ann Emerg Med 1996;27:305-8.

5. Worster A, Bledsoe RD, Cleve P, et al. Reassessing the methods of medical record review studies in emergency medicine research. Ann Emerg Med 2005;45:448-51.

6. Gustavii B. How to write and illustrate a scientific paper. Cambridge (UK): Cambridge University Press, 2003.

7. Sheen AP. Breathing life into medical writing: a handbook. St. Louis (MO): Mosby; 1982.

8. Browner WS. Publishing and presenting clinical research. 2nd ed. Philadelphia (PA): Lippincott Williams \& Wilkins; 2006.

9. Huth EJ. Writing and publishing in medicine. 3rd ed. Baltimore (MD): Williams \& Wilkins, 1999.

Correspondance à : cjem@ caep.ca 